\title{
Understanding local structure and redox chemistry of metal ions in nanoporous catalysts by X-ray absorption spectroscopy
}

\author{
E. Borfecchia \\ Dept. of Chemistry, University of Turin, INSTM reference center and NIS center, Via P. Giuria 7, 10125, Turin (Italy) \\ elisa.borfecchia@unito.it
}

X-ray absorption spectroscopy (XAS) has imposed as a powerful method to track structural and chemical dynamics of metal ions hosted in nanoporous frameworks, such as zeolites and metal-organic frameworks (MOFs), for selective redox catalysis applications [1]. Analysis of the XANES and EXAFS regions offers a highly complementary view with respect to diffraction-based methods guaranteeing a unique sensitivity to the local electronic and structural properties of metal centers. These are often disorderly distributed in the crystalline matrix, and occur as dynamic mixtures of different species, responding to the physico-chemical environment while undergoing a rich redox chemistry mediated by host-guest interactions. Continuous instrumental developments at synchrotron sources today enable in situ/operando XAS studies at high time and energy resolution, allowing to monitor such dynamic systems with unprecedented accuracy [1]. In this contribution, the potential of these methods, empowered by advanced data analysis strategies and synergic integration with multi-technique laboratory characterization and computational modelling, will be exemplified by selected research results.

A first example will focus on the $\mathrm{Cu}$-exchanged chabazite $(\mathrm{Cu}-\mathrm{CHA})$ zeolite, currently representing the catalyst of choice for deNO applications in the automotive sector via $\mathrm{NH}_{3}$-assisted Selective Catalytic Reduction [2]. Here, the potential of Multivariate Curve Resolution (MCR) of time-resolved XANES datasets, quasi-simultaneous XANES/PXRD, and EXAFS Wavelet Transform analysis will be highlighted, to accurately quantify condition/composition-dependent $\mathrm{Cu}$-speciation in CHA zeolites and therein establish robust structure-activity relationships, essential to design improved catalysts. A second case study will consider local structural and chemical transformations of Pt ions in Pt-functionalized UiO-67 MOFs [3], tracked by parametric refinement of time-resolved operando EXAFS under conditions yielding either isolated $\mathrm{Pt}^{\mathrm{II}}$ sites anchored to the MOF framework (potentially interesting for $\mathrm{C}-\mathrm{H}$ bond activation) or very small $\mathrm{Pt}^{0}$ nanoparticles inside the MOF cavities (potentially interesting for hydrogenation reactions).

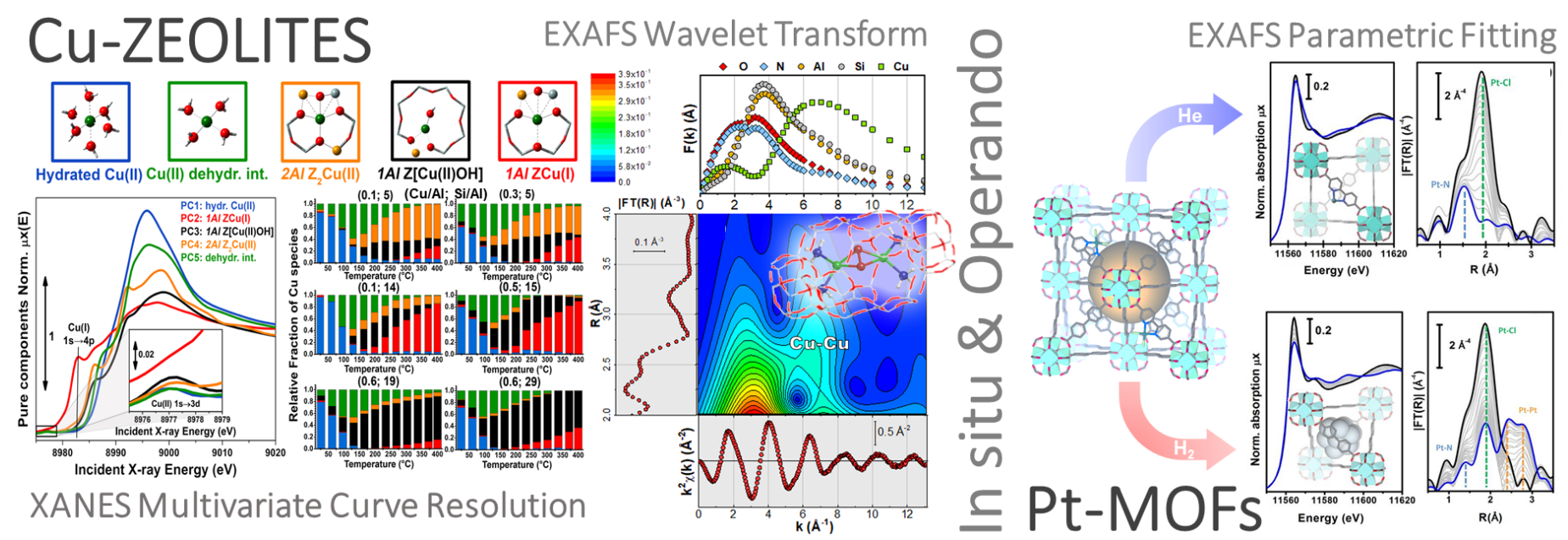

Figure 1. Selected examples and relevant keywords illustrating the potential of advanced analysis methods of in situ/operando XAS data to track structural/chemical dynamics of metal ions hosted in zeolites (left) and MOFs (right) for redox catalysis applications.

[1] S. Bordiga et al., Chem. Rev. 2013, 113, 1736. C. Garino et al., Coord. Chem. Rev. 2014, 277-278, 130. E. Borfecchia et al., Chem. Soc. Rev. 2018, 47, 8097.

[2] A. Martini et al, Chem. Sci. 2017, 8, 6836. C. W. Andersen, et al., Angew. Chem. Int. Edit. 2017, 56, 10367. K. A. Lomachenko et al., J. Am. Chem. Soc. 2016, 138, 12025. C. Negri et al., J. Am. Chem. Soc. 2020, 142, 15884.

[3] S. Øien, et al., Chem. Mater. 2015, 27, 1042. L. Braglia, et al., Phys. Chem. Chem. Phys. 2017, 19, 27489. L. Braglia, et al., Faraday Discuss. 2017, 201, 265.

Keywords: XAS, XANES, EXAFS, MCR, Wavelet Transform, in situ/operando, zeolites, MOFs, selective redox catalysis

This contribution is dedicated to Prof. Carlo Lamberti, gratefully remembering his brilliant guidance and endless curiosity. 